\title{
Coronavirus Derived Expression Systems
} Progress and problems

\author{
${ }^{1}$ LUIS ENJUANES, ISABEL SOLA, FERNANDO ALMAZAN, ANDER \\ IZETA, JOSE M. GONZALEZ, AND SARA ALONSO \\ ${ }^{1}$ Centro Nacional de Biotecnologia, CSIC, Department of Molecular and Cell Biology, \\ Campus Universidad Autónoma, Cantoblanco, 28049 Madrid, Spain
}

\section{INTRODUCTION}

Coronaviruses have several advantages to be used as vectors over other viral expression systems: (i) coronaviruses are single-stranded RNA viruses that replicate within the cytoplasm without a DNA intermediary, making unlikely the integration of the virus genome into the host cell chromosome; (ii) these viruses have the largest RNA genome known having in principle room for the insertion of large foreign genes; (iii) since coronaviruses in general infect the mucosal surfaces, both respiratory and enteric, they may be used to induce a strong secretory immune response; (iv) the tropism of coronaviruses may be modified by the manipulation of the spike (S) protein allowing the engineering of the tropism of the vector; and, (v) nonpathogenic coronavirus strains infecting most species of interest are available to develop expression systems.

Two types of expression vectors have been developed based on coronavirus genomes (Fig. 1), one requires two components (helper dependent) and the other, a single genome that is modified either by targeted recombination or by engineering a cDNA encoding an infectious RNA. 
This review will focus on the advantages and limitations of these novel coronavirus expression systems, and the attempts to increase their expression levels by studying the influence of the transcription regulatory sequences (TRSs).

A

HELPER DEPENDENT EXPRESSION SYSTEM

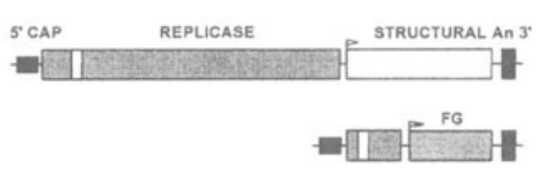

B

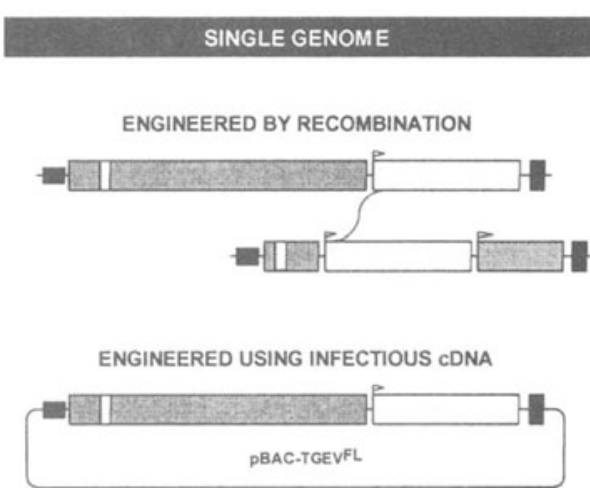

Figure 1. Coronavirus derived expression systems. A. Helper dependent expression system based in two components, the helper virus and a minigenome carrying the foreign gene (FG). An, poly A. B. Single genome engineered either by targeted recombination of by using an infectious coronavirus $\mathrm{cDNA}$ clone $\left(\mathrm{pBAC}-\mathrm{TGEV}^{\mathrm{FL}}\right)$ derived from TGEV genome.

\section{HELPER DEPENDENT EXPRESSION SYSTEMS}

The helper dependent expression systems have been developed using members of the three groups of coronaviruses (Fig. 2). Coronavirus derived minigenomes have a theoretical cloning capacity close to $25 \mathrm{~kb}$, since minigenome RNAs of about $3 \mathrm{~kb}$ are efficiently amplified and packaged by the helper virus and the virus genome has about $30 \mathrm{~kb}$. This is in principle the largest cloning capacity for a vector based on RNA virus genomes.

Most of the initial work required for the development of helper dependent expression systems based on coronaviruses has been done with MHV defective RNAs (Fig. 2) (Liao, Zhang, and Lai, 1995; Lin and Lai, 1993; Zhang et al., 1997). Three heterologous genes have been expressed using MHV system, chloramphenicol acetyltransferase (CAT), hemagglutininesterase (HE), and interferon $\gamma$ (Fig. 2). After intracerebral inoculation of the virus vectors expressing CAT and HE into mice, HE- or CAT-specific subgenomic mRNAs were detected in the brain at days 1 and 2 p.i. but not later, indicating that the genes in the defective genome (DI) vector were expressed only in the early stage of viral infection (Zhang et al., 1998). CAT expression has also been shown using an internal ribosomal entry site sequence (IRES) of encephalomyocarditis virus (Lin and Lai, 1993) (Fig. 2). The murine IFN- $\gamma$ gene was secreted into culture medium as early as 6 $\mathrm{hr}$ post-transfection and reached a peak level at $12 \mathrm{hr}$ post-transfection. 
Infection of susceptible mice with DI RNA producing IFN- $\gamma$ caused significantly milder disease, accompanied by less virus replication than that caused by virus containing a control DI vector (Lai et al., 1997; Zhang et al., 1997).
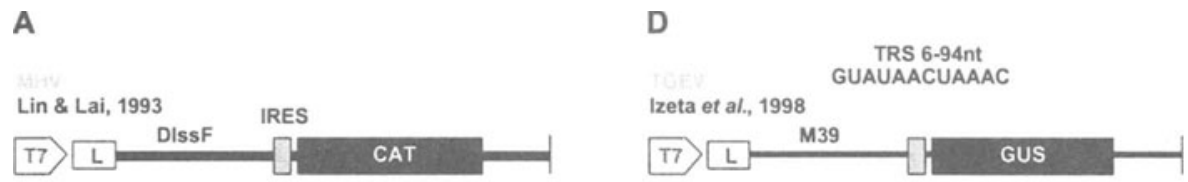

B

E
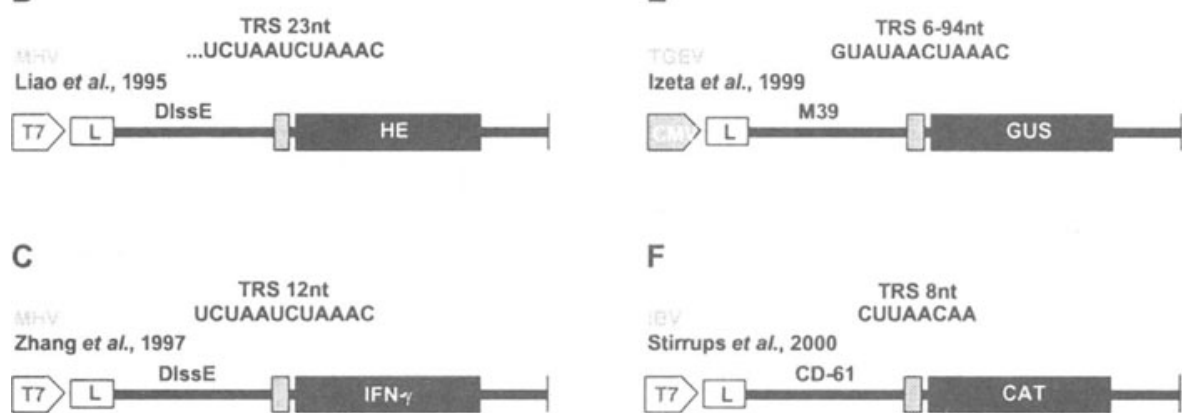

Figure 2. Summary of helper-dependent expression systems based on coronavirus derived minigenomes. A, B, and C. expression modules based MHV on minigenomes DIssF and DIssE cloned after the T7 bacteriophage polymerase (T7), used to express chloramphenicol acetyltransferase (CAT), hemagglutinin-esterase (HE) or interferon- $\gamma$ using either an IRES (A), or transcription regulatory sequences (B and C). D, E. expression modules based on the TGEV derived minigenome M39 used to express the $\beta$-glucuronidase (GUS); the minigenome is cloned after the CMV promoter (Izeta et al., 1998). F. Expression module based on the IBV derived minigenome CD-61 used to express CAT.

Group 1 coronaviruses such as transmissible gastroenteritis virus (TGEV) has also been used to express foreign proteins (Fig. 2). The vector included a two step amplification (Dubensky et al., 1996), by cloning a cDNA copy of M39 minigenome after the CMV promoter. Minigenome RNAs are first amplified in the nucleus by the cellular RNA pol II, then the RNAs are translocated into the cytoplasm where they are amplified by the viral replicase of the helper virus. The smallest TGEV derived minigenome (M33) that was replicated by the helper virus and efficiently packaged was $3.3 \mathrm{~kb}$ (Izeta et al., 1999; Méndez et al., 1996). In addition to GUS, the ORF5 of the porcine respiratory and reproductive syndrome virus (PRRSV) has been expressed (Alonso et al., 2000b).

The HCoV-229E has also been used to express new subgenomic mRNAs, although it has not been applied to the expression of a foreign protein (Thiel, Siddell, and Herold, 1998). In addition, a defective RNA (CD-61) derived from the Beaudette strain of the IBV virus (Penzes et al., 
1994; Penzes et al., 1996) was used as an RNA vector for the expression of CAT (Fig. 2) (Stirrups et al., 2000).

A helper dependent expression system has recently been described based on arteriviruses, closely related to coronaviruses (Molenkamp et al., 2000). Using equine arteritis virus (EAV) minigenomes of $3.8 \mathrm{~kb}$ the CAT reporter gene has been produced. The smallest defective RNA replicated by the helper virus had a 3.0-kb length, but this RNA was not packaged.

The expression levels have not been quantified in terms of protein mass for MHV expression systems. Expression levels of CAT between 1-2 $\mu \mathrm{g}$ per $10^{6}$ cells have been described using IBV minigenomes. The highest amount of protein ( 1 to $8 \mu \mathrm{g}$ of GUS per $10^{6}$ cells) have been obtained using a two step amplification system based on TGEV derived minigenomes with optimized TRSs (Alonso et al., 2000a; Izeta et al., 1999). These protein levels are similar to those described for vectors based on other positive strand RNA viruses such as poliovirus and the Venezuelan encephalitis virus (VEEV) $\left(4 \mu \mathrm{g}\right.$ per $\left.10^{6}\right)$, but still lower than the expression levels described for Sindbis virus: $50 \mu \mathrm{g}$ per $10^{6}$ (Agapov et al., 1998) and SFV: $80-300 \mu \mathrm{g}$ per $10^{6}$ cells (DiCiommo and Bremner, 1998; Liljeström and Garoff, 1991).

\section{SINGLE GENOME CORONAVIRUS VECTORS}

\subsection{Vectors constructed by targeted recombination}

Reverse genetics has been possible by targeted recombination between a helper virus and either non-replicative or replicative coronavirus derived RNAs (Fig. 3A) (Masters, 1999). Targeted recombination has been mediated by one or two cross-overs. Changes were introduced within the $\mathrm{S}$ gene that modified MHV pathogenicity (Leparc-Goffart et al., 1998). The gene encoding green fluorescent protein (GFP) was inserted into MHV between genes $\mathrm{S}$ and $\mathrm{E}$ by targeted recombination, resulting in the creation of the largest known RNA viral genome (Fischer et al., 1997). Mutations have also been created by targeted mutagenesis within the $\mathrm{E}$ and the $\mathrm{M}$ genes showing the crucial role of these genes in assembly (de Haan et al., 1998; Fisher and Goff, 1998).

Targeted recombination mediated by two cross-overs allowed the replacement of the $S$ gene of a respiratory strain of TGEV by the $S$ gene of enteric TGEV leading to the isolation of viruses with a modified tropism and virulence (Sánchez et al., 1999). A new strategy for the selection of TGEV recombinants was based in the elimination of the parental replicative virus by its simultaneous neutralization with two mAbs (Fig. 3B) (Sola et al., 2000). 


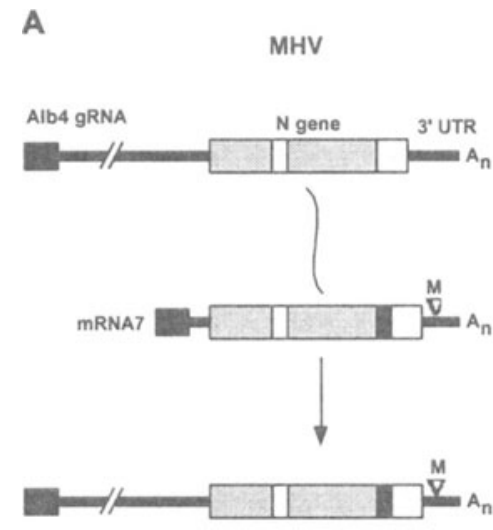

Koetzner et al., 1992

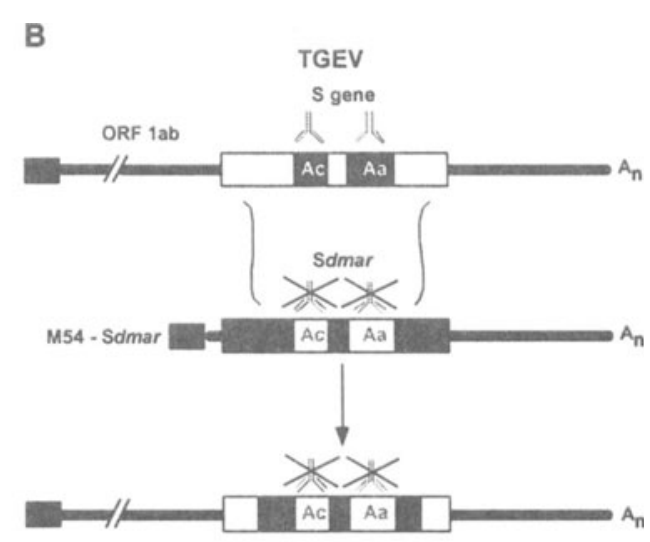

Sola et al., 2000

Figure 3. Single genome expression based on the engineering of coronavirus minigenomes by targeted recombination. A. Basic scheme of targeted recombination in MHV. The black boxe indicates the approximate location of the $\mathrm{N}$ gene region (87nt) that is deleted in the Alb4 mutant. M, insertion of $5 \mathrm{nt}$ used as a genetic marker (M). B. Targeted recombination within the $\mathrm{S}$ gene of TGEV and a minigenome carrying the information for an S gene with three nucleotide mutations (Sdmar) that allow the escaping from the neutralization by two $\mathrm{mAbs}$ specific for antigenic sub-sites Ac and Aa of S protein.

The frequencies of the targeted recombination event in MHV and TGEV recombination were found higher than the standard prediction for the recombination frequency of a multiple cross-over. This frequency was expected to be the product of the frequencies of the individual recombination events. This suggests that the alignment of two templates is the rate-limiting event in recombination and, once this has been achieved, the barrier to multiple crossovers may be only marginally higher than that for single crossovers (Masters, 1999; Sola et al., 2000).

\subsection{Coronavirus vectors derived from an infectious cDNA clone}

The construction of a full-length genomic cDNA clone could considerably improve the genetic manipulation of coronaviruses. Now, for the first time, the construction of an infectious TGEV cDNA clone has been possible (Almazan et al., 2000). To obtain an infectious cDNA three strategies have been combined (i) the construction of the full-length cDNA was started from a DI that was stably and efficiently replicated by the helper virus (Izeta et al., 1999; Méndez et al., 1996). Using this DI, the fulllength genome was completed and the performance of the enlarged genome was checked after each step. This approach allowed for the identification of a cDNA fragment that was toxic to the bacterial host. This finding was used 


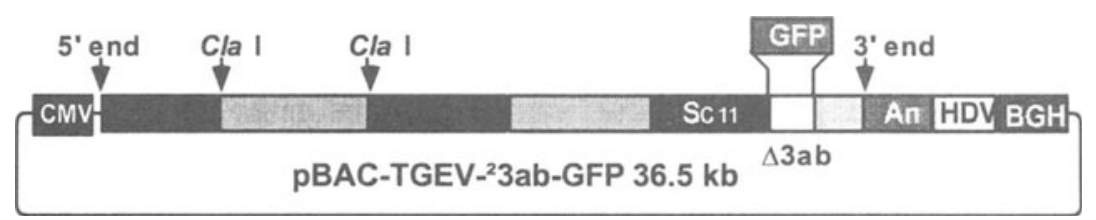

Figure 4. Expression of GFP using an infectious TGEV cDNA clone. Genes $3 \mathrm{a}$ and $3 \mathrm{~b}$ were deleted in the TGEV infectious cDNA, cloned in a bacterial artificial chromosome (BAC), leading to a replication competent cDNA (pBAC-TGEV- $\triangle 3 \mathrm{ab}-\mathrm{GFP})$. GFP $(0.72 \mathrm{~kb})$ was inserted within the position of the deleted genes after the TRS of gene $3 \mathrm{a}$. High $\left(>40 \mu \mathrm{g} / 10^{6}\right.$ cells) GFP expression levels were obtained. CMV, immediate-early cytomegalovirus promoter. GFP, green fluorescent protein. SC11, S gene of PUR-C11 TGEV strain. An, poly A. HDV, hepatitis delta-virus ribozyme. BGH, bovine growth hormone termination and polyadenylation signals.

to advantage by reintroducing the toxic fragment into the viral cDNA in the last cloning step; (ii) in order to express the long coronavirus genome, and to add the 5' cap, a two-step amplification system that couples transcription in the nucleus from the CMV promoter, with a second amplification in the cytoplasm, driven by the viral replicase, was used; and, (iii) to increase viral cDNA stability within bacteria, the cDNA was cloned as a bacterial artificial chromosome (BAC), that produces only one or two plasmid copies per cell. The full-length cDNA was divided into two plasmids because their fusion into one reduced the stability of the cDNA. One plasmid contained all virus sequences except for a fragment Cla I to Cla I of about $5 \mathrm{~kb}$ that was included within a second BAC. A fully functional infectious cDNA clone, leading to a virulent virus able to infect both the enteric and respiratory tracts, was engineered by inserting the $\mathrm{Cla} I$ fragment into the rest of the TGEV cDNA sequence (Fig. 4). Using the TGEV cDNA, the GFP gene was cloned by replacing the non-essential $3 a$ and $3 b$ genes, leading to an engineered genome with high stability (Fig. 4) (Sola et al., 2000).

The theoretical cloning capacity for an expression system based on a single coronavirus genome like TGEV may be around $3 \mathrm{~kb}$ taking into account that: (i) the non-essential $3 \mathrm{a}$ and $3 \mathrm{~b}$ genes $(\sim 1.0 \mathrm{~kb})$ have been deleted; (ii) the standard S gene can been replaced by that of PRCV mutants with a deletion of $0.67 \mathrm{~kb}$; and (iii) both DNA and RNA viruses may accept genomes with sizes up to $105 \%$ of the wild type genome (Afanasiev et al., 1999; Bett, Prevec, and Graham, 1993; Parks and Graham, 1997). The present cloning capacity of the coronavirus vectors is within the range expected, since other RNA virus vectors, such as those derived from the Sindbis virus and VEEV, with a genome of around $12 \mathrm{~kb}$, accept stable inserts of about $1 \mathrm{~kb}$ in size (Bredenbeek and Rice, 1992; Caley et al., 1997). 


\section{REGULATION OF TRANSCRIPTION}

\subsection{Introduction}

Coronavirus RNA synthesis occurs in the cytoplasm via a negativestrand RNA intermediate. Both genome-size and subgenomic negativestrand RNAs, which correspond in number of species and size to those of the virus-specific mRNAs have been detected. The two transcription models compatible with most of the experimental data are leader-primed transcription and discontinuous transcription during negative-strand RNA synthesis (Lai, 1998). Recently, more experimental evidence is being generated supporting the second model (Baric and Yount, 2000; Sawicki and Sawicki, 1990; Sethna, Hung, and Brian, 1989; van Marle et al., 1999).

Viral RNA replication and transcription may involve cellular proteins taken from the translation machinery of host cells (Lai, 1998). Two cellular hnRNPs, polypyrimidine tract-binding protein (PTB) and hnRNP Al, bind to the transcription regulatory sequences (TRSs) of MHV RNA and may participate in its transcription (Li et al., 1999; Li et al., 1997).

Many factors including RNA primary and secondary structure, RNAprotein and protein-protein interactions could influence mRNA abundance. One of these factors, the nature of the TRSs and the extent of their complementarity with the 3 ' end sequence of the leader, may be the most relevant, and is discussed below.

The TRSs include the core sequence (CS), previously named intergenic sequence (IG), that is a short conserved sequence element upstream of the transcription units. Because the leader-mRNA junction occurs within the $\mathrm{CS}$, this motif or its minus-sense counterpart (cCS) are considered to be crucial for mRNA synthesis. The nature of the cCS probably influences transcription throughout its potential basepairing with the leader 3' end. According to this model the cCS should act as a classical promoter where transcription is initiated. Alternatively this sequence may slow down or even detach the transcriptase complex, according to the discontinuous transcription during negative-strand RNA synthesis model.

Most of the information on coronavirus transcription has been generated using helper dependent expression systems based on minigenomes encoding new subgenomic mRNAs. The CSs of coronaviruses belonging to groups I (hexameric 5'-CUAAAC-3') and II (heptameric 5'-UCUAAAC-3') share homology, whereas the CS of coronaviruses belonging to group III, like that of IBV have the most divergent sequence (5'-UAACAA-3').

\subsection{Extent of basepairing and mRNA levels}

The potential basepairing between the 3' end of the leader and the cCS differs slightly among the different coronavirus genes. For MHV, the extent 
of the basepairing ranges from 9 to 18 basepairs and these CSs were sufficient to direct subgenomic DI RNA synthesis (Joo and Makino, 1992; van der Most, De Groot, and Spaan, 1994; van der Most and Spaan, 1995).

In MHV cCS strength is affected only slightly when a single nucleotide is mutated (Joo and Makino, 1992; van der Most, De Groot, andSpaan, 1994). Exceptionally, substitutions in some positions result in a more than ten-fold reduction of transcription. These data suggest that transcription initiation requires a duplex of a minimal stability. Extending this basepairing does not increase $\mathrm{CCS}$ strength.

Using TGEV derived RNA minigenomes, we have shown that the CS sequence 5'-CUAAAC-3' is required and sufficient for high expression levels (Alonso et al., 2000a). Similarly, in IBV, expression of the reporter gene was under one canonical octameric IBV CS sequence 5'CUUAACAA-3' (Stirrups et al., 2000). In Arteriviruses, it has also been shown that subgenomic mRNA (sgmRNA) synthesis requires base-pairing between the leader 3 '-end and the cCS. EAV CS consists of pentanucleotide 5'-UCAAC-3' (van Marle et al., 1999). Thus, expression both in coronavirus and arteriviruses can be driven by a TRS with less than $18 \mathrm{nt}$ in size.

The sequences flanking the consensus core sequence 3'-UCUAAAC-5' affected the efficiency of subgenomic DI RNA transcription (Joo and Makino, 1992; Makino and Joo, 1993; Makino, Joo, and Makino, 1991; van der Most, De Groot, andSpaan, 1994). The insertion of a 12 nt sequence including the 5'-UCUAAAC-3' CS at different locations of the DI RNA resulted in different efficiencies of subgenomic DI synthesis as a consequence of the flanking sequences in each position, and not due to the location of the $12 \mathrm{nt}$ sequence on the DI genome.

In TGEV, the absence of the core CS (5'-CUAAAC-3') or the deletion of the $\mathrm{U}$ within ORF $3 \mathrm{~b}$ CS led to the complete abrogation of mRNA transcription (Alonso et al., 2000a). The insertion of the hexameric 5'CUAAAC-3' restored expression levels more than 400-fold above background. The addition of 5' upstream sequences flanking the core CS from the TGEV N gene, led to an increase in transcription of up to 10-fold, indicating the benefit of TRSs of larger ( $88 \mathrm{nt})$ size.

The sequences 3' downstream to the core CS of seven viral genes ( $\mathrm{S}, 3 \mathrm{a}$, $3 \mathrm{~b}, \mathrm{E}, \mathrm{M}, \mathrm{N}$, and 7) have sizes ranging from 3 to $37 \mathrm{nt}$. Expression modules in which the 5' flanking sequence was kept constant, and the 3' CS flanking sequences were provided by each of the seven $3^{\prime}$ flanking sequences of the viral genes, led to similar expression levels, with the exception of that from ORF 3a. This construct gave expression levels 5- to 10-fold lower. Thus, there was no correlation between the length of the 3' flanking sequences and the expression level (Alonso et al., 2000a), but careful selection of the 3 ' flanking sequences is recommended to optimize mRNA levels. 
More data is required to clarify the role of baseparing between the leader $3^{\prime}$ end and the CS, and also the relevance of the primary or secondary sequence of the TRSs.

\subsection{CS copy number effect on transcription}

Insertion of two to three CS copies within a defective RNA using MHV, $\mathrm{BCoV}$ and IBV resulted in the decreased transcription of the larger mRNA (Joo and Makino, 1995; van Marle et al., 1995). In all cases a negative effect on the transcription of upstream CSs by the downstream ones was observed. When several CSs are inserted in tandem, transcription preferentially occurred at the 3'-most TRS (Krishnan, Chang, and Brian, 1996; Stirrups et al., 2000).

\subsection{Influence of the insertion site}

Using a TGEV derived helper dependent expression system, the reporter gene GUS was inserted at different nucleotide distances from the $5^{\prime}$. The expression levels increased from the $5^{\prime}$ to $3^{\prime}$ end by one thousand-fold (Alonso et al., 2000a).

In a systematic study using MHV, a $0.4 \mathrm{~kb}$ region including a TRS of 12 nt flanked by $0.2 \mathrm{~kb}$ from upstream and downstream was inserted throughout the sequence at seven different positions within a $2.2 \mathrm{~kb}$ minigenome (Jeong et al., 1996). The position of the insert along the minigenome did not influence the mRNA expression level. In the experiments performed with TGEV, the insertion site close to the $5^{\prime}$ end probably have affected essential primary or secondary structures required for minigenome replication, thus reducing the significance of this result in relationship to the insertion site. Furthermore, no difference in expression levels was observed with the MHV system, in which the flanking sequences were kept constant for all insertion sites, suggesting that the location of the insertion site per se does not necessarily affect transcription levels and that the differences observed with TGEV were mostly due to the CS-flanking sequences.

\subsection{Expression system stability and insert size}

Expression from MHV defective RNAs of CAT, HE and murine IFN- $\gamma$ genes using was not observed beyond passages 2,3 and 4, respectively. Using minigenomes derived from TGEV and IBV expression was more stable but highly dependent on the nature of the heterologous gene that was expressed. GUS or CAT expression with TGEV or IBV derived minigenomes, respectively, was observed for about ten passages (Alonso et al., 2000a; Stirrups et al., 2000). In general, the insertion of a heterologous 
gene such as GUS into TGEV derived minigenomes led to a 50-fold reduction in the levels of minigenome RNA replication (Alonso et al., 2000a). The limited stability of the helper dependent expression systems is most likely due to the foreign gene, since TGEV minigenomes in the absence of the heterologous gene are efficiently rescued for at least 30 passages (Izeta et al., 1999; Méndez et al., 1996). The recombination frequency in MHV, TGEV, and IBV may be inversely proportional to the stability of the recombinants expressing a foreign gene. The stability of the MHV expression system is the lowest, probably because of the higher recombination frequency within MHV (Lai, 1996).

The stability of the expression system is conditioned by the type of polymerases involved in the amplification of the minigenome and in the transcription of the mRNA (Agapov et al., 1998). For in vitro transcribed minigenome RNAs, the accumulation of mutations with T7 DNAdependent RNA-polymerase is $10^{-4}$ to $10^{-5}$ (Boyer, Bebenek, and Kunkel, 1992). Minigenome RNAs transcribed by viral RNA-dependent RNApolymerases will have an accumulation of mutations of $10^{-3}$ to $10^{-4}$ (de Mercoyrol et al., 1992). An improvement in expression stability should be observed by using expression systems initiated by DNA transfection, such as those based on the expression of the minigenomes under CMV promoter since an eukaryotic RNA polymerase II has an estimated error frequency of $5 \times 10^{-6}$ (de Mercoyrol et al., 1992).

\section{CONCLUSIONS}

Both helper-dependent expression systems, based on two components, and single genomes constructed by targeted recombination or by using an infectious cDNA have been developed. The sequences that regulate transcription have been characterized. Expression of high amounts of heterologous antigens ( 1 to $8 \mu \mathrm{g} / 10^{6}$ cells) have been achieved, and the expression levels have been maintained for around 10 passages. These expression levels should be sufficient to elicite protective immune responses.

Single genome coronavirus vectors have been constructed efficiently expressing a foreign gene such as GFP. Thus, a new avenue with high potential has been opened for coronaviruses which have unique properties, such as a long genome size and enteric tropism, that makes them of high interest as expression vectors for vaccine development and gene therapy. The possibility of engineering the tissue and species tropism will make coronavirus very flexible expression systems, since the same vector could be modified to target expression to different organs and animal species, including humans. 


\section{ACKNOWLEDGEMENTS}

This work has been supported by grants from the Comision Interministerial de Ciencia y Tecnología (CICYT), La Consejería de Educación y Cultura de la Comunidad de Madrid, and Fort Dodge Veterinaria from Spain, and the European Communities (Key Action 2: Infectious Diseases). JO and JMG received fellowships from the Department of Research and Technology. ISG, FA and PB, received contracts from the European Union Biotechnology, FAIR and Key Action 2: Infectious Diseases. AI and SA received fellowships from the Department of Education, University and Research of the Gobierno Vasco; JMS, received a fellowship from the Veterinary College of the Community of Madrid, CR received a fellowship from the Spanish Department of Health.

\section{REFERENCES}

Afanasiev, B. N., Ward, T. W., Beaty, B. J., and Carlson, J. O., 1999, Transduction of Aedes aegypti mosquitoes with vectors derived from Aedes densovirus. Virology 257: 62-72.

Agapov, E. V., Frolov, I., Lindenbach, B. D., Pragai, B. M., Schlesinger, S., and Rice, C. M., 1998, Noncytopathic Sindbis virus RNA vectors for heterologous gene expression. Proc. Natl. Acad. Sci. USA 95: 12989-12994.

Almazan, F., González, J. M., Pénzes, Z., Izeta, A., Calvo, E., Plana-Durán, J., and Enjuanes, L., 2000, Engineering the largest RNA virus genome as an infectious bacterial artificial chromosome. Proc. Natl. Acad. Sci. USA 97: 5516-5521.

Alonso, S., Izeta, A., Sola, I., and Enjuanes, L., 2000a, Transcription regulatory sequences in transmissible gastroenteritis coronavirus. Submitted.

Alonso, S., Sola, I., Wege, H., Teifke, J., and Enjuanes, L., 2000b, Heterologous gene expression in tissue culture and in vivo using a transmissible gastroenteritis coronavirus helper dependent system. Submitted.

Baric, R. S., and Yount, B., 2000, Subgenomic negative-strand RNA function during mouse hepatitis virus infection. J. Virol. 74: 4039-4046.

Bett, A. J., Prevec, L., and Graham, F. L., 1993, Packaging capacity and stability of human adenovirus type 5 vectors. J. Virol. 67: 5911-5921.

Boyer, J. C., Bebenek, K., and Kunkel, T. A., 1992, Unequal human immunodeficiency virus type 1 reverse transcriptase error rates with RNA and DNA templates. Proc. Natl. Acad. Sci. USA 89: 6919-6923.

Bredenbeek, P. J., and Rice, C. M., 1992, Animal RNA virus expression systems. Semin. Virol. 3: 297-310.

Caley, I. J., Betts, M. R., Irlebeck, D. M., Davis, N. L., Swanstrom, R., Frelinger, J. A., and Johnston, R. E., 1997, Humoral, mucosal, and cellular immunity in response to a human immunodeficiency virus type 1 immunogen expressed by a venezuelan equine encephalitis virus vaccine vector. J. Virol. 71: 3031-3038.

de Haan, C. A. M., Kuo, L., Masters, P. S., Vennema, H., and Rottier, P. J. M., 1998, Coronavirus particle assembly: primary structure requirements of the membrane protein. J. Virol. 72: 6838-6850.

de Mercoyrol, L., Corda, Y., Job, C., and Job, D., 1992, Accuracy of wheat-germ RNA polymerase II. General enzymatic properties and effect of template conformational transition from right-handed B- DNA to left-handed Z-DNA. Eur. J. Biochem. 206: 49-58. 
DiCiommo, D. P., and Bremner, R., 1998, Rapid, high level protein production using DNAbased Semliki Forest virus vectors. J. Biol. Chem. 17: 18060-18066.

Dubensky, T. W., Driver, D. A., Polo, J. M., Belli, B. A., Latham, E. M., Ibanez, C. E., Chada, S., Brumm, D., Banks, T. A., Mento, S. J., Jolly, D. J., and Chang, S. M. W., 1996, Sindbis virus DNA-based expression vectors: utility for in vitro and in vivo gene transfer. J. Virol. 70: 508-519.

Fischer, F., Stegen, C. F., Koetzner, C. A., and Masters, P. S., 1997, Analysis of a recombinant mouse hepatitis virus expressing a foreign gene reveals a novel aspect of coronavirus transcription. J. Virol. 71: 5148-5160.

Fisher, J., and Goff, S. P., 1998, Mutational analysis of stem-loops in the RNA packaging signal of the Moloney murine leukemia virus. Virology 244: 133-145.

Izeta, A., Sánchez, C. M., Smerdou, C., Méndez, A., Alonso, S., Balasch, M., Plana-Durán, J., and Enjuanes, L., 1998, The spike protein of transmissible gastroenteritis coronavirus controls the tropism of pseudorecombinant virions engineered using synthetic minigenomes. Adv. Exp. Med. Biol. 440: 207-214.

Izeta, A., Smerdou, C., Alonso, S., Penzes, Z., Méndez, A., Plana-Durán, J., and Enjuanes, L., 1999, Replication and packaging of transmissible gastroenteritis coronavirusderived synthetic minigenomes. J. Virol. 73: 1535-1545.

Jeong, Y. S., Repass, J. F., Kim, Y.-N., Hwang, S.-M., and Makino, S., 1996, Coronavirus transcription mediated by sequences flanking the transcription consensus sequence. Virology 217: 311-322.

Joo, M., and Makino, S., 1992, Mutagenic analysis of the coronavirus intergenic consensus sequence. J. Virol. 66: 6330-6337.

Joo, M., and Makino, S., 1995, The effect of two closely inserted transcription consensus sequences on coronavirus transcription. J. Virol. 69: 272-280.

Krishnan, R., Chang, R. Y., and Brian, D. A., 1996, Tandem placement of a coronavirus promoter results in enhanced mRNA synthesis from the downstream-most initiation site. Virology 218: 400-405.

Lai, M. M. C., 1996, Recombination in large RNA viruses: coronaviruses. Semin. Virol. 7: 381-388.

Lai, M. M. C., 1998, Cellular factors in the transcription and replication of viral RNA genomes: a parallel to DNA-dependent RNA transcription. Virology 244: 1-12.

Lai, M. M. C., Zhang, X., Hinton, D., and Stohlman, S., 1997, Modulation of mouse hepatitis virus infection by defective-interfering RNA-mediated expression of viral proteins and cytokines. J. Neurovirol. 3:(Supp. 1) S33-S34.

Leparc-Goffart, I., Hingley, S. T., Chua, M. M., Phillips, J., Lavi, E., and Weiss, S. R., 1998, Targeted recombination within the spike gene of murine coronavirus mouse hepatitis virus-A59: Q159 is a determinant of hepatotropism. J. Virol. 72: 9628-9636.

Li, H.-P., Huang, P., Park, S., and Lai, M. M. C., 1999, Polypyrimidine tract-binding protein binds to the leader RNA of mouse hepatitits virus and serves as a regulator of viral transcription. J. Virol. 73: 772-777.

Li, H.-P., Zhang, X., Duncan, R., Comai, L., and Lai, M. M. C., 1997, Heterogeneous nuclear ribonucleoprotein $\mathrm{Al}$ binds to the transcription-regulatory region of mouse hepatitis virus RNA. Proc. Natl. Acad. Sci. USA 94: 9544-9549.

Liao, C. L., Zhang, X., and Lai, M. M. C., 1995, Coronavirus defective-interfering RNA as an expression vector: the generation of a pseudorecombinant mouse hepatitis virus expressing hemagglutinin-esterase. Virology 208: 319-327.

Liljeström, P., and Garoff, H., 1991, A new generation of animal cell expression vectors based on the Semliki Forest virus replicon. Biotechnology 9: 1356-1361.

Lin, Y. J., and Lai, M. M. C., 1993, Deletion mapping of a mouse hepatitis virus defective interfering RNA reveals the requirement of an internal and discontiguous sequence for replication. J. Virol. 67: 6110-6118.

Makino, S., and Joo, M., 1993, Effect of intergenic consensus sequence flanking sequences on coronavirus transcription. J. Virol. 67: 3304-3311. 
Makino, S., Joo, M., and Makino, J. K., 1991, A system for study of coronavirus messenger RNA synthesis: a regulated, expressed subgenomic defective interfering RNA results from intergenic site insertion. J. Virol. 65: 6031-6041.

Masters, P. S., 1999, Reverse genetics of the largest RNA viruses. Adv. Virus Res. 53: 245264.

Méndez, A., Smerdou, C., Izeta, A., Gebauer, F., and Enjuanes, L., 1996, Molecular characterization of transmissible gastroenteritis coronavirus defective interfering genomes: packaging and heterogeneity. Virology 217: 495-507.

Molenkamp, R., Rozier, B. C. D., Greve, S., Spaan, W. J. M., and Snijder, E. J., 2000, Isolation and characterization of an arterivirus defective interfering RNA genome. $J$. Virol. 74: 3156-3165.

Parks, R. J., and Graham, F. L., 1997, A helper-dependent system for adenovirus vector production helps define a lower limit for efficient DNA packaging. J. Virol. 71: 32933298.

Penzes, Z., Tibbles, K., Shaw, K., Britton, P., Brown, T. D. K., and Cavanagh, D., 1994, Characterization of a replicating and packaged defective RNA of avian coronavirus infectious bronchitis virus. Virology 203: 286-293.

Penzes, Z., Wroe, C., Brown, T. D. K., Britton, P., and Cavanagh, D., 1996, Replication and packaging of coronavirus infectious bronchitis virus defective RNAs lacking a long open reading frame. J. Virol. 70: 8660-8668.

Sánchez, C. M., Izeta, A., Sánchez-Morgado, J. M., Alonso, S., Sola, I., Balasch, M., PlanaDurán, J., and Enjuanes, L., 1999, Targeted recombination demonstrates that the spike gene of transmissible gastroenteritis coronavirus is a determinant of its enteric tropism and virulence. J. Virol. 73: 7607-7618.

Sawicki, S. G., and Sawicki, D. L., 1990, Coronavirus transcription: subgenomic mouse hepatitis virus replicative intermediates function in RNA synthesis. J. Virol. 64: 10501056.

Sethna, P. B., Hung, S.-L., and Brian, D. A., 1989, Coronavirus subgenomic minus-strand RNAs and the potential for mRNA replicons. Proc. Natl. Acad. Sci. USA 86: 56265630 .

Sola, I., Izeta, A., González, J. M., and Enjuanes, L., 2000, Tissue specific expression into the mucosal surface using a single genome vector based on recombinant coronaviruses. Submitted.

Stirrups, K., Shaw, K., Evans, S., Dalton, K., Casais, R., Cavanagh, D., and Britton, P., 2000, Expression of reporter genes from the coronavirus infectious bronchitis virus defective RNA CD-61. J. Gen. Virol. In press: 000-000.

Thiel, V., Siddell, S. G., and Herold, J., 1998, Replication and transcription of HCV 229E replicons. Adv. Exp. Med. Biol. 440: 109-114.

van der Most, R. G., De Groot, R. J., and Spaan, W. J. M., 1994, Subgenomic RNA synthesis directed by a synthetic defective interfering RNA of mouse hepatitis virus: a study of coronavirus transcription initiation. J. Virol. 68: 3656-3666.

van der Most, R. G., and Spaan, W. J. M., 1995, Coronavirus replication, transcription, and RNA recombination. In "The Coronaviridae" (S. G. Siddell, Ed.), pp. 11-31. Plenum Press, New York.

van Marle, G., Dobbe, J. C., Gultyaev, A. P., Luytjes, W., Spaan, W. J. M., and Snijder, E. J., 1999, Arterivirus discontinuous mRNA transcription is guided by base pairing between sense and antisense transcription-regulating sequences. Proc. Nat. Acad. Sc. USA 96: 12056-12061.

van Marle, G., Luytjes, W., Van der Most, R. G., van der Straaten, T., and Spaan, W. J. M., 1995, Regulation of Coronavirus mRNA transcription. J. Virol. 69: 7851-7856.

Zhang, X., Hinton, D. R., Cua, D. J., Stohlman, S. A., and Lai, M. M. C., 1997, Expression of interferon- $\gamma$ by a coronavirus defective-interfering RNA vector and its effect on viral replication, spread, and pathogenicity. Virology 233: 327-338.

Zhang, X., Hinton, D. R., Park, S., Parra, B., Liao, C.-L., and Lai, M. M. C., 1998, Expression of hemagglutinin/esterase by a mouse hepatitis virus coronavirus defectiveinterfering RNA alters viral pathogenesis. Virology 242: 170-183. 